\title{
Partially ionized atmospheres of neutron stars with strong magnetic fields
}

\author{
A.Y. Potekhin ${ }^{\text {a,* }}$, Dong Lai ${ }^{\mathrm{b}}$, G. Chabrier ${ }^{\mathrm{c}}$, W.C.G. Ho ${ }^{\mathrm{d}, 1}$ \\ ${ }^{a}$ Ioffe Physico-Technical Institute, 194021 St. Petersburg, Russia \\ ${ }^{\mathrm{b}}$ Department of Astronomy, Cornell University, Ithaca, NY 14853, USA \\ ${ }^{\mathrm{c}}$ Ecole Normale Supérieure de Lyon, CRAL (UMR CNRS No. 5574), \\ 46 allée d'Italie, 69364 Lyon, France \\ ${ }^{\mathrm{d}}$ Kavli Institute for Particle Astrophysics and Cosmology, Stanford University, \\ Stanford, CA 94309, USA
}

\begin{abstract}
We construct hydrogen atmosphere models for strongly magnetized neutron stars in thermodynamic equilibrium, taking into account partial ionization. The presence of bound states affects the equation of state, absorption coefficients, and polarizability tensor of a strongly magnetized plasma. Therefore the partial ionization influences the polarization vectors and opacities of normal electromagnetic waves, and thus the spectra of outgoing radiation. Here we review a model suitable for the most typical neutron-star atmospheres and focus on the problems that remain to be solved for its extension to other atmospheric parameters.
\end{abstract}

Key words: stellar atmospheres, neutron stars, strong magnetic fields

\section{Introduction}

Thermal emission from neutron stars (NSs) may potentially be used to directly measure the NS surface magnetic field, temperature, and composition, achieve a more complete understanding of the evolution of the NSs, and constrain the properties of matter and physical processes under extreme conditions. It was realized long ago that a NS atmosphere model should properly include a

\footnotetext{
* Corresponding author. Tel.: +7-812-2479180; fax: +7-812-5504890.

Email address: palex@astro.ioffe.ru (A.Y. Potekhin).

1 Hubble fellow.
} 
strong magnetic field and partial ionization (see, e.g., Pavlov et al., 1995, for an early review). Models of fully ionized NS atmospheres with strong magnetic fields were constructed by several research groups (e.g., Shibanov et al., 1992; Zane et al., 2000; Ho and Lai, 2003, and references therein). The most recent papers highlighted the effects that may be important for the atmospheres of magnetars: the ion cyclotron feature (Ho and Lai, 2001; Zane et al., 2001) and vacuum polarization effects, including a conversion of the normal modes of radiation propagating in the magnetized atmosphere (Ho and Lai, 2003; Lai and Ho, 2002, 2003).

Early considerations of partial ionization in the magnetized NS atmospheres (Miller 1992; Rajagopal et al. 1997; also reviewed briefly by Zavlin \& Pavlov 2002) were not very reliable because of oversimplified treatments of atomic physics and nonideal plasma effects in strong magnetic fields. At the typical NS atmosphere parameters, the effects of thermal motion of the bound species are important. In the 1990s, binding energies and radiative transition rates with allowance for the motion effects in strong magnetic fields have been calculated for the H atom (Potekhin, 1994; Potekhin and Pavlov, 1997). Recently these atomic data have been implemented in calculations of thermodynamic functions (Potekhin et al., 1999; Potekhin and Chabrier, 2003, 2004), radiative opacities (Potekhin and Chabrier, 2003, 2004), and spectra (Ho et al., 2003 ) of the partially ionized $\mathrm{H}$ atmospheres of the NSs. Some results have been presented at the previous Cospar meeting (Ho et al., 2004a). Now our atmosphere model has been complemented by the effects of the bound states on the polarization properties of the strongly magnetized plasma (Potekhin et al., 2004). Below we briefly summarize the results that allow us to calculate realistic X-ray spectra of thermal radiation from hydrogen NS atmospheres with magnetic fields $B \sim 10^{12}-10^{14} \mathrm{G}$ and effective temperatures $T \gtrsim 10^{5.5} \mathrm{~K}$, and outline the problems that remain to be addressed at other atmospheric parameters and compositions.

\section{The atmosphere model}

We use the equation of state (EOS) for H in strong magnetic fields (Potekhin et al., 1999) based on the free-energy minimization method, which ensures the thermodynamic consistency and allows one to determine number fractions of chemical species, required for opacity calculations. The model takes into account all available theoretical results on the moving $\mathrm{H}$ atoms and nonideal Coulomb plasmas in the magnetic fields. This EOS has been tabulated and employed for calculation of opacities for astrophysical use (Potekhin and Chabrier, 2003, 2004).

It is well known that under typical conditions (e.g., far from the resonances) 

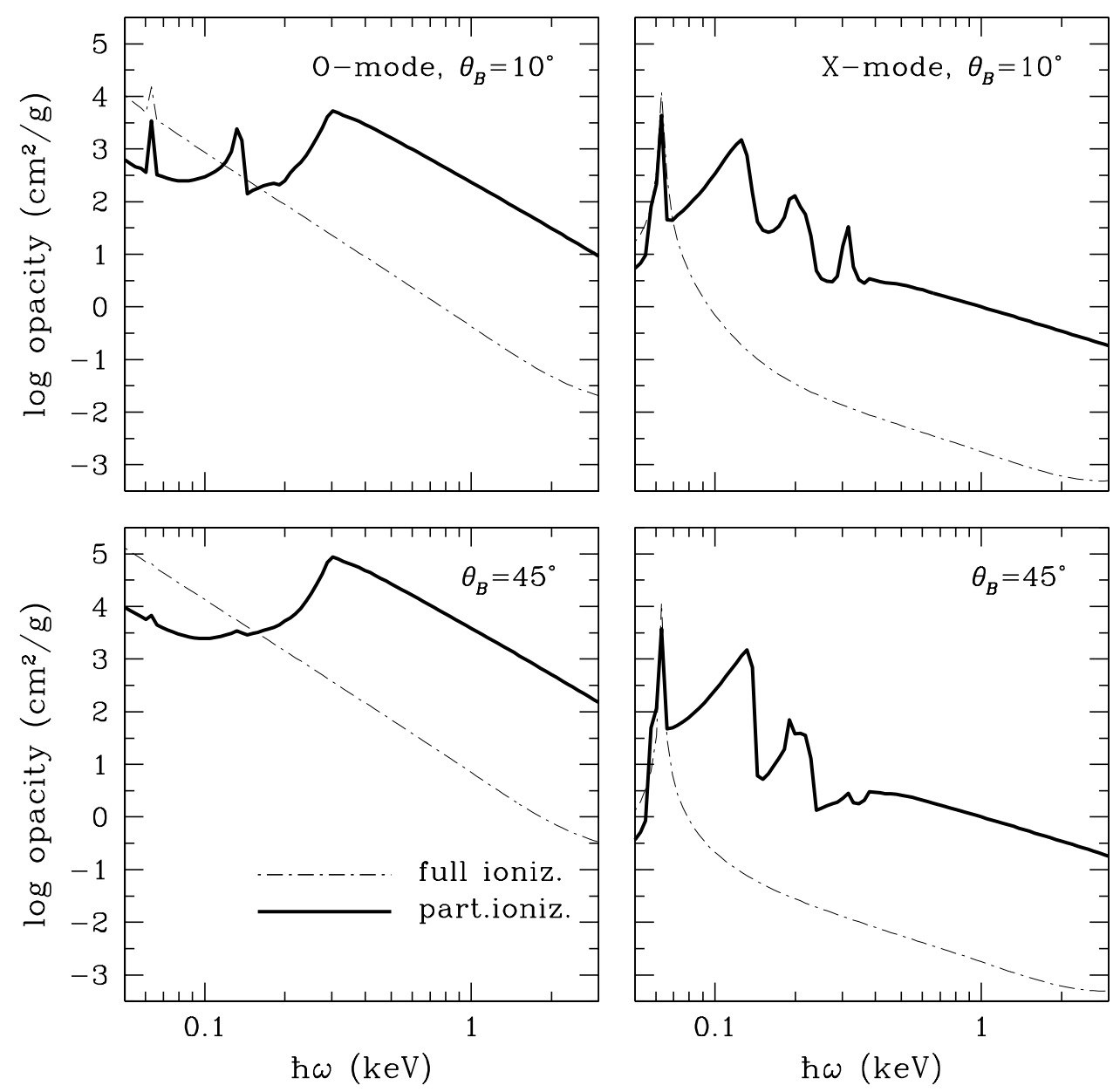

Fig. 1. Opacities $\kappa_{j}$ (Eq. [1]) in the O-mode (left panels) and X-mode (right panels) versus photon energy in the hydrogen plasma at $B=10^{13} \mathrm{G}, T=10^{5.5} \mathrm{~K}$, and $\rho=1 \mathrm{~g} \mathrm{~cm}^{-3}$, for $\theta_{B}=10^{\circ}$ (upper panels) and $45^{\circ}$ (lower panels). Solid lines: a self-consistent calculation for a partially ionized plasma (70\% of neutrals); dot-dashed lines: the model of full ionization.

radiation propagates in a magnetized plasma in the form of two normal modes, called the extraordinary $(\mathrm{X})$ and the ordinary $(\mathrm{O})$. The opacity in the mode $j(j=\mathrm{X}, \mathrm{O})$ depends on the photon frequency $\omega$, magnetic field $B$, density $\rho$, temperature $T$, and the angle $\theta_{B}$ between the magnetic field and propagation direction. It can be written as

$$
\kappa_{j}\left(\omega, \theta_{B}\right)=\sum_{\alpha=-1}^{1}\left|e_{\alpha}^{j}\left(\omega, \theta_{B}\right)\right|^{2} \hat{\kappa}_{\alpha}(\omega),
$$

where $e_{\alpha}^{j}(\alpha=-1,0,1)$ are the cyclic coordinates of the polarization vectors of the normal modes, and the quantities $\hat{\kappa}_{\alpha}(\alpha=-1,0,1)$ do not depend on $\theta_{B}$. 
Potekhin and Chabrier (2003) calculated $\hat{\kappa}_{\alpha}(\omega)$ and evaluated $\kappa_{j}\left(\omega, \theta_{B}\right)$ using the polarization vectors of the normal modes in the fully ionized plasma, Such a calculation (dubbed "hybrid" in Potekhin et al. 2004) was employed in our previous model of partially ionized hydrogen atmospheres of the NSs with strong magnetic fields (Ho et al., 2003, 2004a).

In the new model (Potekhin et al., 2004), we take into account the influence of the bound species on the polarization vectors of the normal modes, making use of the Kramers-Kronig relation ${ }^{2}$ between the imaginary and real parts of the plasma polarizability. Thus the calculation of the polarization vectors and opacities of the normal modes has become self-consistent. The calculations of thermal spectra of the NSs show that such self-consistent treatment is necessary if the number fraction of the bound states exceeds several percent. In Fig. 1 we compare radiative opacities calculated with and without allowance for the bound species for one particular set of plasma parameters, typical near the radiative surface of a moderately cool neutron star with magnetic field $B=10^{13} \mathrm{G}$, for two $\theta_{B}$ values. In the case shown in the figure, the neutral fraction is $70 \%$, thus the self-consistent treatment of the opacities is important.

\section{Conclusion and unsolved problems}

The constructed atmosphere models allow us to calculate realistic spectra of thermal X-ray radiation from $\mathrm{H}$ atmospheres of the NSs with $10^{12} \mathrm{G} \lesssim B \lesssim$ $10^{14} \mathrm{G}$ and $T \gtrsim 10^{5.5} \mathrm{~K}$. Examples of these spectra are presented elsewhere (Potekhin et al., 2004). There remain the following unsolved problems that prevent us from making similarly reliable calculations beyond these limits.

1. Although the H EOS and opacities have been calculated for $B$ up to $10^{15} \mathrm{G}$ and implemented in the atmosphere model (Ho et al., 2003, 2004a), the calculated spectra at $B \gtrsim 10^{14} \mathrm{G}$ depend on the adopted model of mode conversion due to the vacuum resonance and on description of propagation of photons with frequencies below the plasma frequency. Both these problems have not been definitely solved. Their solution is also important for modeling the lowfrequency (UV and optical) tail of the spectrum.

2. At lower $T$ or higher $B, \mathrm{H}$ atoms recombine in $\mathrm{H}_{n}$ molecules and eventually form the condensed phase (Lai, 2001, and references therein). Corresponding quantum-mechanical data are very incomplete.

$\overline{2}$ Previously this relation has been used by Bulik \& Pavlov (1996) for a neutral gas of $\mathrm{H}$ atoms in a strong magnetic field. 
3. At $10^{9} \mathrm{G} \lesssim B \lesssim 10^{11} \mathrm{G}$, transition rates of the moving $\mathrm{H}$ atom have not been calculated previously because of their complexity. The first calculation of the energy spectrum appropriate in this range of $B$ has been published when the present paper was in preparation (Lozovik and Volkov, 2004).

4. At present it is not possible to calculate accurate atmospheric spectra at $B \gtrsim 10^{12} \mathrm{G}$ for chemical elements other than hydrogen, because of the importance of the effects of finite nuclear mass in the strong field regime. Apart from the $\mathrm{H}$ atom, these effects have been calculated only for the He atom at rest (Al-Huiai and Schmelcher, 2003a, b) and for the $\mathrm{He}^{+}$ion (at only one value of $B$, Bezchastnov et al. 1998).

5. A more rigorous treatment of radiative transfer in the atmosphere requires solving the transfer equations of the Stokes parameters (see, e.g., Lai and Ho 2003 for the cases of fully ionized atmospheres). However, since the nonorthogonal features of the modes due to neutral species are diminished by the centerof-mass motion, the effect is expected to be small.

Finally, let us note that the atmosphere model presented here, together with a model of radiation from the condensed magnetic surface van Adelsberg et al., 2004), has been successfully used for fitting the spectrum of the isolated neutron star RX J1856.5-3754 (Ho et al., 2004b).

Acknowledgements. A.P. acknowledges the hospitality of the Astronomy Department of Cornell University and the theoretical astrophysics group at the Ecole Normale Supérieure de Lyon. The work of A.P. is supported in part by RFBR grants 02-02-17668 and 03-07-90200, and RLSS grant 1115.2003.2. D.L. is supported in part by NSF grant AST 0307252, NASA grant NAG 5-12034, and SAO grant TM4-5002X. W.H. is supported by NASA through Hubble Fellowship grant HF-01161.01-A awarded by STScI, which is operated by AURA, Inc., for NASA, under contract NAS 5-26555.

\section{References}

Al-Hujaj, O.-A., Schmelcher, P. Helium in superstrong magnetic fields. Phys. Rev. A 67, 023403 (11 p.), 2003a.

Al-Hujaj, O.-A., Schmelcher, P. Electromagnetic transitions of the helium in superstrong magnetic fields. Phys. Rev. A 68, 053403 (7 p.), 2003b.

Bezchastnov, V.G., Pavlov, G.G., Ventura, J. Discrete eigenstates of the $\mathrm{He}^{+}$ ion moving in a strong magnetic field. Phys. Rev. A 58, 180-185, 1998.

Bulik, T., \& Pavlov, G. G. Polarization modes in a strongly magnetized hydrogen gas. Astrophys. J. 469, 373-387, 1996.

Ho, W.C.G., Lai, D. Atmospheres and spectra of strongly magnetized neutron stars. Mon. Not. R. Astron. Soc. 327, 1081-1096, 2001. 
Ho, W.C.G., Lai, D. Atmospheres and spectra of strongly magnetized neutron stars. II. The effect of vacuum polarization. Mon. Not. R. Astron. Soc. 338, 233-252, 2003.

Ho, W.C.G., Lai, D., Potekhin, A.Y., Chabrier, G. Atmospheres and spectra of strongly magnetized neutron stars. III. Partially ionized hydrogen models. Astrophys. J. 599, 1293-1301, 2003.

Ho, W.C.G., Lai, D., Potekhin, A.Y., Chabrier, G. Atmospheres of magnetized neutron stars: vacuum polarization and partially ionized models. Adv. Sp. Res. 33, 537-541, 2004a.

Ho, W.C.G., Kaplan, D.L., Chang, P., van Adelsberg, M. Modeling the spectrum of the isolated neutron star RX J1856.5-3754 with thin hydrogen atmospheres. Astrophys. J., submitted, 2004b.

Lai, D. Matter in strong magnetic fields. Rev. Mod. Phys. 73, 629-661, 2001.

Lai, D., Ho, W.C.G. Resonant conversion of photon modes due to vacuum polarization in a magnetized plasma: implications for X-ray emission from magnetars. Astrophys. J. 566, 373-377, 2002.

Lai, D., Ho, W.C.G. Transfer of polarized radiation in strongly magnetized plasmas and thermal emission from magnetars: effect of vacuum polarization. Astrophys. J. 588, 962-974, 2003.

Lozovik, Yu.E., Volkov, S.Yu. Hydrogen atom moving across a magnetic field. Phys. Rev. A 70, 023410 (8 p.), 2004.

Miller, M.C. Model atmospheres for neutron stars. Mon. Not. R. Astron. Soc. 255, 129-145, 1992.

Pavlov, G.G., Shibanov, Yu.A., Zavlin, V.E., Meyer, R.D. Neutron star atmospheres, in: Alpar, M.A., Kiziloğlu, Ü., van Paradijs, J. (Eds.), The Lives of the Neutron Stars. Kluwer, Dordrecht, pp. 71-90, 1995.

Potekhin, A.Y. Structure and radiative transitions of the hydrogen atom moving in a strong magnetic field. J. Phys. B: At. Mol. Opt. Phys. 27, 1073-1090, 1994.

Potekhin, A.Y., Chabrier, G. Equation of state and opacities for hydrogen atmospheres of neutron stars with strong magnetic fields. Astrophys. J. 585, 955-974, 2003.

Potekhin, A.Y., Chabrier, G. Equation of state and opacities for hydrogen atmospheres of magnetars. Astrophys. J. 600, 317-323, 2004.

Potekhin, A.Y., Pavlov, G.G. Photoionization of hydrogen in atmospheres of magnetic neutron stars. Astrophys. J. 483, 414-425, 1997.

Potekhin, A.Y., Chabrier, G., Shibanov, Yu.A. Partially ionized hydrogen plasma in strong magnetic fields. Phys. Rev. E 60, 2193-2208, 1999.

Potekhin, A.Y., Lai, D., Chabrier, G., Ho, W.C.G. Electromagnetic polarization in partially ionized plasmas with strong magnetic fields and neutron star atmosphere models. Astrophys. J. 612, 1034-1043, 2004.

Rajagopal M., Romani R., Miller M.C. Magnetized iron atmospheres for neutron stars. Astrophys. J. 479, 347-356, 1997.

Shibanov, Yu.A., Pavlov, G.G., Zavlin, V.E., Ventura, J. Model atmospheres and radiation of magnetic neutron stars. I - The fully ionized case. Astron. 
Astrophys. 266, 313-320, 1992.

van Adelsberg, M., Lai, D., Potekhin, A.Y. Radiation from condensed surface of magnetic neutron stars. Astrophys. J., submitted, 2004 (astro-ph/0406001)

Zane, S., Turolla, R., Treves, A. Magnetized atmospheres around neutron stars accreting at low rates. Astrophys. J. 537, 387-395, 2000.

Zane, S., Turolla, R., Stella, L., Treves, A. Proton cyclotron features in thermal spectra of ultramagnetized neutron stars. Astrophys. J. 560, 384-389, 2001.

Zavlin, V.E., Pavlov, G.G. Modeling neutron star atmospheres, in: Becker, W., Lesch, H., Trümper, J. (Eds.), Proceedings of the 270. WE-Heraeus Seminar on Neutron Stars, Pulsars, and Supernova Remnants, MPE Report 278, pp. 263-272, 2002. 